\title{
Retraction and Correction
}

\section{RETRACTION}

\section{GENETICS, SOCIAL SCIENCES}

Retraction for "PKNOX2 gene is significantly associated with substance dependence in European-origin women," by Xiang Chen, Kelly Cho, Burton H. Singer, and Heping Zhang, which published online August 31, 2009, in Proc Natl Acad Sci USA (10.1073/pnas.0908521106).

The authors wish to retract this paper because its publication violates the Gene Environment Association Studies Genes and Environment Initiative Study of Addiction: Genetics and Environment (SAGE) dataset's embargo policy. The SAGE data access agreement states that investigators agree not to submit findings of the SAGE dataset(s) for publication until September 23, 2009. The authors sincerely apologize for this violation of SAGE policy.

\section{Xiang Chen \\ Kelly Cho \\ Burton H. Singer Heping Zhang}

www.pnas.org/cgi/doi/10.1073/pnas.0910252106

\section{CORRECTION}

\section{NEUROSCIENCE}

Correction for "Stomach ghrelin-secreting cells as foodentrainable circadian clocks," by Joseph LeSauter, Nawshin Hoque, Michael Weintraub, Donald W. Pfaff, and Rae Silver, which appeared in issue 32, August 11, 2009, of Proc Natl Acad Sci USA (106:13582-13587; first published July 24, 2009; 10.1073/ pnas.0906426106).

The authors note that on page 13583, in the legend for Fig. 2, an equation appeared incorrectly. The figure and its corrected legend appear below. Additionally, in Fig. $3 A$ on page 13584 , the panels labeled "Ghrelin" and "PER1" appeared incorrectly. The corrected figure and its legend appear below. These errors do not affect the conclusions of the article.
A
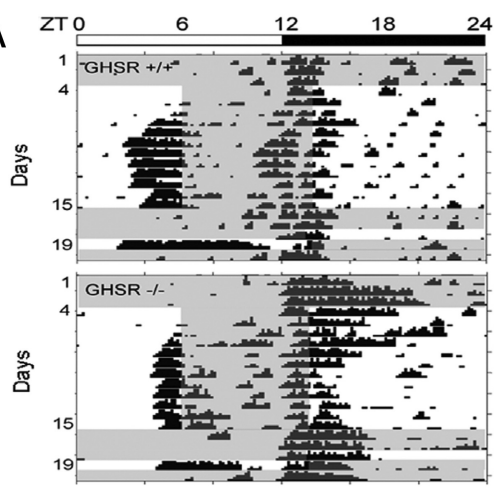

$\mathrm{B}$
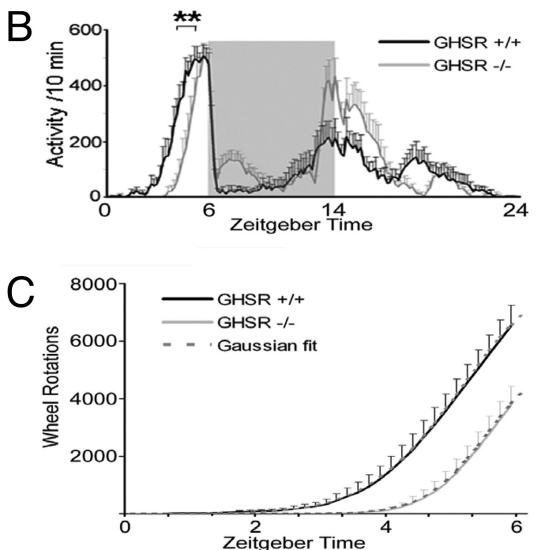

Fig. 2. Running wheel behavior of wild-type and $\mathrm{GHSR}^{-1-}$ mice during ad libitum feeding, food restriction, and food deprivation conditions. $(A)$ The bar above the actograms shows the light-dark cycle; time of food availability is shown in gray. Actograms depict activity of representative $\mathrm{GHSR}^{+/+}$and $\mathrm{GHSR}^{-1-}$ mice during ad libitum feeding (days 1-4), food restriction ZT6-ZT14 (days 4-15), ad libitum food availability (days 15-18), and food deprivation (day 19). (B) Group activity profiles show the amount of wheel running during the last 7 days of restricted feeding in $\mathrm{GHSR}^{+1+}$ (black) and $\mathrm{GHSR}^{-1-}$ (gray) mice. The data are plotted in 10-min bins (mean $\pm \mathrm{SEM}$ ). **, $P=0.002$, difference between $\mathrm{GHSR}^{+/+}$and $\mathrm{GHSR}^{-1-}$ in onset time of activity. (C) Line graph of cumulative wheel-running activity (mean \pm SEM) from lights on (ZTO) to time of food presentation (ZT6) shows that $\mathrm{GHSR}^{-1-}$ mice (solid gray line) ran $42.4 \%$ less than $\mathrm{GHSR}^{+1+}$ (solid black line) mice. Superimposed are the curves derived from the Gaussian function $f(x)=e^{-x^{2} / 2} / \sqrt{2 \pi}$ (dashed lines). (D) The anticipation ratios during 7 days of restricted feeding (Top) and the persistence ratio (Middle) on the day of food deprivation are shown for $\mathrm{GHSR}^{-1-}$ (gray bars) and control GHSR ${ }^{+/+}$(black bars) mice, with significant differences between groups. (Bottom) Daily activity during the period of food restriction. *, $P=0.02 ; * *, P=0.01$. 